\title{
Modes of structurally chiral lasers
}

\author{
René D. M. Topf* and Martin W. McCall \\ The Blackett Laboratory, Department of Physics, Imperial College London, Prince Consort Road, London SW7 2AZ, United Kingdom
}

(Received 24 April 2014; published 12 November 2014)

\begin{abstract}
We employ coupled wave theory to enumerate the lasing modes of structurally chiral lasers. The elliptical modes are shown to be fundamentally distinct from those of a scalar distributed feedback laser. High threshold modes are shown to lase with the opposite chirality as the active medium, in contrast to their low-threshold counterparts that lase with the same chirality as the active medium. The lasing mode structure suggests the intriguing possibility of dynamically changing the polarization handedness of a chiral laser, as well as the possibility of lasing within the forbidden band-gap region. These observations arise from the fundamental interplay between the distributed chirality-preserving reflections within the active medium and the localized chirality-reversing reflections at the medium's boundaries.
\end{abstract}

DOI: 10.1103/PhysRevA.90.053824

PACS number(s): 42.55.Tv, 42.70.Df, 42.70.Qs, 42.81.Gs

\section{INTRODUCTION}

Photons, the mediating bosons of the electromagnetic field, are inherently chiral, carrying a spin of $\pm \hbar$. Understanding chiral lasing sources is therefore a natural objective that can influence several photonic fields, from single-photon quantum information processing [1] to RealD 3D digital stereoscopic projection technology [2]. Moreover, ultrathin, highly versatile, wideband-tunable chiral sources $[3,4]$ are promising candidates for replacing vertical-cavity surfaceemitting lasers and dye lasers in applications such as medical imaging and display technology [5-7].

Micrometer-sized chiral sources are being inkjet printed onto plastic films to produce cheap, disposable, tunable, flexible laser arrays that can emit RGB and white light for use in displays and hologram projection. Low-cost, compact, widely tunable chiral laser prototypes have been created, which are continuously tunable from 450 to $850 \mathrm{~nm}$ and have a slope efficiency of $60 \%-70 \%$ [5,8-10]. Self-assembling chiral fiber lasers are being created, which range in size from a couple of micrometres to about $100 \mu \mathrm{m}$ in diameter and can grow several centimeters long. These fiber lasers can be manipulated with laser tweezers in order to build soft photonic circuits [7].

A clear understanding of the modes issued by structurally chiral sources is necessary for the continued development of these promising devices. In this paper we develop the vector coupled wave theory (CWT) of structurally chiral lasers and predict a class of polarized modes that are contrahanded to the active medium's structural chirality. These modes enrich the lasing structure of chiral lasers, as they can lase inside the chiral photonic band gap and open up the possibility for fast polarization switching.

Scalar distributed feedback (DFB) lasers were first analyzed by Kogelnik and Shank [11]. Kopp et al. observed chiral DFB lasing at the band edge [12] (where the photon dwell time is largest) in dye-doped cholesteric liquid crystals, a gain enhancement technique first proposed by Dowling et al. [13]. It has since been demonstrated that chiral lasers

\footnotetext{
*rene.topf08@imperial.ac.uk
}

can combine microcavities with large output powers, large coherence areas, and high tunability throughout the visible spectrum $[14,15]$.

\section{CHIRAL LASER THEORY}

In contrast to scalar DFB lasers, the active medium of a structurally chiral laser is a birefringent dielectric with a spatially periodic rotation of the transverse principal axes, as depicted in Fig. 1. Structurally chiral media (SCMs), such as chiral sculptured thin films [16] or cholesteric liquid crystals [17], provide polarization-specific DFB; circularly polarized light copolarized with the medium helicity experiences an index modulation of amplitude equal to the local birefringence $\delta n$, whereas the orthogonal circular polarization propagates as if through a uniform medium of average refractive index $\bar{n}$. For simplicity, we consider a right-handed structure for the remainder of the paper.

Axial propagation of the transverse electric field $\mathbf{E}_{\perp}$ is described by the Helmholtz equation

$$
d^{2} \mathbf{E}_{\perp} / d z^{2}+k_{0}^{2} \boldsymbol{\epsilon}_{\perp} \cdot \mathbf{E}_{\perp}=0,
$$

where $\epsilon_{\perp}$ is the transverse permittivity tensor and $k_{0}$ is the free space wave number. Coupled wave theory is used to rewrite the Helmholz equations as first-order coupled differential equations of the axially propagating forward and backward waves, resolved on a circular basis as

$$
\frac{d}{d z}\left[\begin{array}{c}
E_{L}^{+} \\
E_{L}^{-} \\
E_{R}^{+} \\
E_{R}^{-}
\end{array}\right]=\left[\begin{array}{cccc}
i k & 0 & 0 & 0 \\
0 & -i k & 0 & 0 \\
0 & 0 & i k & i \kappa e^{2 i p z} \\
0 & 0 & -i \kappa e^{-2 i p z} & -i k
\end{array}\right]\left[\begin{array}{c}
E_{L}^{+} \\
E_{L}^{-} \\
E_{R}^{+} \\
E_{R}^{-}
\end{array}\right],
$$

with the real coupling constant $\kappa \approx \pi \delta n / \lambda_{0}$ and $p=2 \pi / L_{p}$. Solving Eq. (2) provides [18]

$$
\left[\begin{array}{c}
E_{L}^{+}(z) \\
E_{L}^{-}(z) \\
E_{R}^{+}(z) \\
E_{R}^{-}(z)
\end{array}\right]=\left[\begin{array}{cccc}
e^{i k z} & 0 & 0 & 0 \\
0 & e^{-i k z} & 0 & 0 \\
0 & 0 & \mathcal{P}^{+} & \mathcal{Q}^{+} \\
0 & 0 & \mathcal{Q}^{-} & \mathcal{P}^{-}
\end{array}\right]\left[\begin{array}{c}
E_{L}^{+}(0) \\
E_{L}^{-}(0) \\
E_{R}^{+}(0) \\
E_{R}^{-}(0)
\end{array}\right],
$$


where

$$
\begin{aligned}
\mathbf{E}_{\perp} & =\frac{E_{L}^{+}+E_{R}^{-}}{\sqrt{2}}\left[\begin{array}{l}
1 \\
i
\end{array}\right]+\frac{E_{L}^{-}+E_{R}^{+}}{\sqrt{2}}\left[\begin{array}{c}
1 \\
-i
\end{array}\right], \\
\mathcal{P}^{ \pm}(z) & =e^{ \pm i p z}\left[\cosh (\Delta z) \pm i \frac{\delta k}{2 \Delta} \sinh (\Delta z)\right], \\
\mathcal{Q}^{ \pm}(z) & = \pm i e^{ \pm i p z} \frac{\kappa}{\Delta} \sinh (\Delta z), \\
\Delta & =\left[\kappa^{2}-(\delta k / 2)^{2}\right]^{1 / 2} .
\end{aligned}
$$

Here $k=\bar{n} k_{0}, \bar{n}$ is complex, and $\delta k=2(k-p)$. In Eq. (3) left circular polarization (LCP) propagates as if in a uniform medium of index $\bar{n}$, while right circular polarization (RCP) propagates as if through a DFB structure of pitch $L_{p} / 2$.

Coupled wave theory has been tested in passive lossy SCMs [18] against the exact analytic solution [16] and was found to be in excellent agreement [11] for our regime, where $\delta n \ll|\bar{n}|$. Further calculations show that the agreement also holds with the introduction of isotropic gain. The exact analytic solution for axial propagation in SCMs involves matrix exponentiation and is not suitable for deriving simple expressions that determine the lasing modes as wave number and gain vary.

The DFB reflection and transmission coefficients of the SCMs at $z=0$ are $r_{c}=-\mathcal{Q}^{-}(L) / \mathcal{P}^{-}(L)$ and $t_{c}=\left[\mathcal{P}^{-}(L)\right]^{-1}$ and correspond to the DFB coefficients for a Bragg grating [19]. Note that $t_{c}(L)=t_{c}(0)$, but that due to phase differences $r_{c}(L)=e^{2 i p L} r_{c}(0)$. The Bragg wavelength, given by $\lambda_{0}^{\mathrm{Br}}=\bar{n} L_{p}$, is half that of an equivalent scalar DFB structure, since optically SCMs repeat every half twist, as seen from the dashed arrow in Fig. 1.

We place the SCM between two uniform media characterized by refractive indices $n_{1}$ and $n_{2}$ (see Fig. 1), so reflections at the interfaces are characterized by Fresnel reflection coefficients $r_{1}=\left(n_{c}-n_{1}\right) /\left(n_{c}+n_{1}\right)$ and $r_{2}=\left(n_{c}-n_{2}\right) /$ $\left(n_{c}+n_{2}\right)$, where $n_{c}=\operatorname{Re}(\bar{n})$. These reflections are chirality

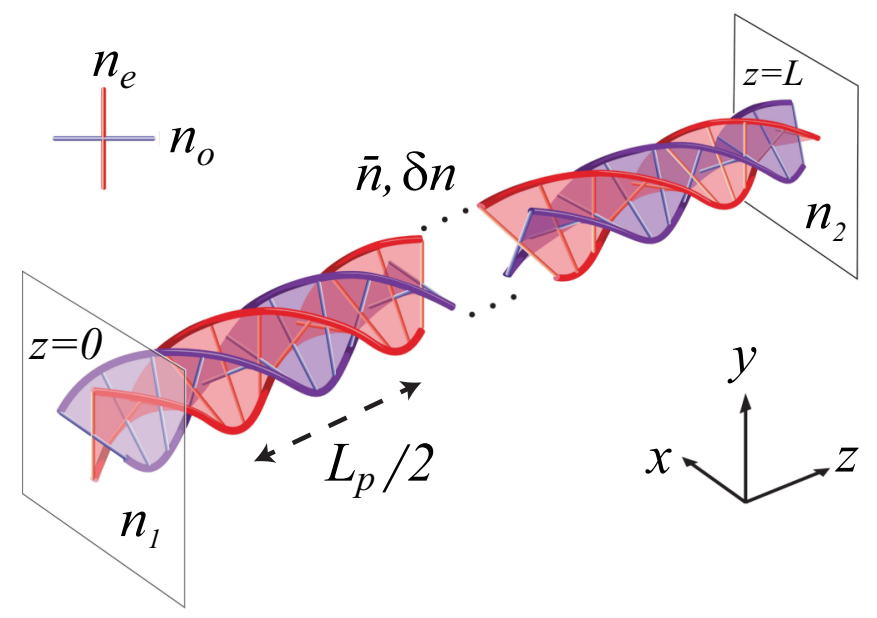

FIG. 1. (Color online) Right-handed SCM with surrounding media of indices $n_{1}$ at $z=0$ and $n_{2}$ at $z=L$. The transverse principal axes are aligned with the coordinate axes at $z=0$ and rotate around the $z$ axis with period $L_{p}$. We show that such right-handed SCMs can support both right-handed and left-handed lasing modes.
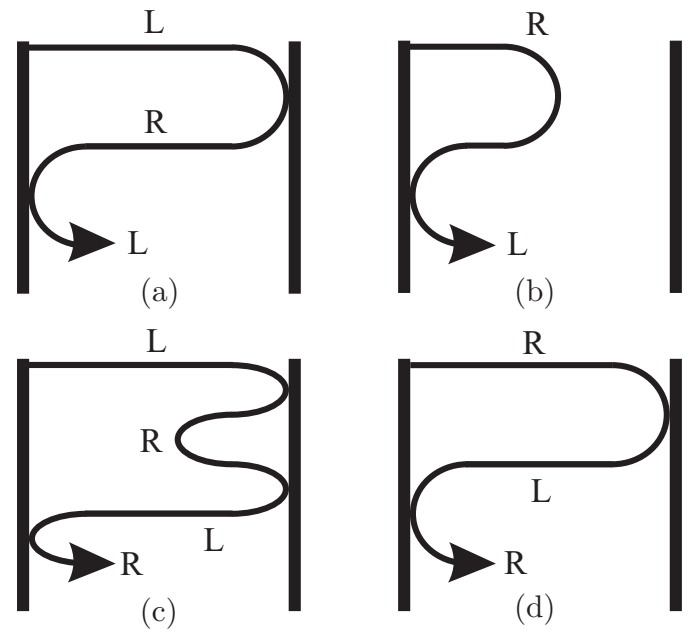

FIG. 2. The four round-trip light paths for circularly polarized light: (a) $a_{L L}=r_{1} r_{2} e^{i k L} t_{c}$, (b) $a_{L R}=r_{1} r_{c}$, (c) $a_{R L}=e^{2 i(k+p) L} r_{1} r_{2}^{2} r_{c}$, and (d) $a_{R R}=e^{i k L} r_{1} r_{2} t_{c}$. Vertical lines represent the SCM boundaries and curved arrows represent the light paths. The letters denote circular polarization state.

reversing and provide a second coupling mechanism that contrasts with the chirality-preserving distributed reflection.

These two coupling mechanisms determine the four possible round-trip paths of circularly polarized light, which are illustrated schematically in Fig. 2. The complex round-trip amplitude $a_{L R}$ [Fig. 2(b)], for example, which represents the amplitude for initial RCP to become LCP after one round-trip, begins with a chirality-preserving distributed reflection $r_{c}$, followed by a chirality-reversing interface reflection $r_{1}$ at the $z=0$ interface. Likewise, $a_{L L}$ [Fig. 2(a)] begins with propagation of LCP through a uniform medium $e^{i k L}$, followed by a chirality-reversing interface reflection $r_{2}$ at $z=L$, followed by RCP transmission $t_{c}$ through the chiral medium, followed by a chirality-reversing interface reflection $r_{1}$ at $z=0$. All round-trip coupling pathways can be constructed from those depicted in Fig. 2.

Using a complex refractive index in CWT enables poles to be determined in the DFB reflectivity as wave number and gain vary [19]. For an index-matched scalar DFB laser the modes were analyzed by Kogelnik and Shank [11] and when boundary reflections were accounted for by Agrawal and Dutta [20].

The modes of a chiral laser are found by setting $z=L$ in Eq. (3), applying the Fresnel equations, and imposing chirality reversal at each boundary. The boundary conditions are therefore

$$
\begin{array}{ll}
E_{L}^{+}(0)=r_{1} E_{R}^{-}(0), & E_{L}^{-}(L)=r_{2} E_{R}^{+}(L), \\
E_{R}^{+}(0)=r_{1} E_{L}^{-}(0), & E_{R}^{-}(L)=r_{2} E_{L}^{+}(L) .
\end{array}
$$

The resulting equations are reduced to the following system for the forward going fields at $z=0$ :

$$
\left[\begin{array}{cc}
r_{1} r_{2} e^{i k L}-\mathcal{P}^{-} & -r_{1} \mathcal{Q}^{-} \\
e^{i k L} r_{2} \mathcal{Q}^{+} & e^{i k L} r_{1} r_{2} \mathcal{P}^{+}-1
\end{array}\right]\left[\begin{array}{l}
E_{L}^{+}(0) \\
E_{R}^{+}(0)
\end{array}\right]=0 .
$$

A chiral laser mode must therefore satisfy

$$
\left(r_{1} r_{2} e^{i k L}\right)^{2} \mathcal{P}^{+}-2 r_{1} r_{2} e^{i k L}+\mathcal{P}^{-}=0,
$$


TABLE I. Data used for calculations.

\begin{tabular}{lcc}
\hline \hline Structural period of SCM & $L_{p}$ & $300 \mathrm{~nm}$ \\
Depth of SCM & $L$ & $6 \mu \mathrm{m}$ \\
Number of half periods & $L / L_{p}$ & 20 \\
Bragg wavelength & $\lambda_{0}^{\mathrm{Br}}$ & $530.4 \mathrm{~nm}$ \\
Average refractive index of SCM (Re) & $n_{c}$ & 1.7680 \\
Chiral birefringence at $\lambda_{0}^{\mathrm{Br}}(\mathrm{Re})$ & $\delta n$ & $0-0.1126$ \\
Refractive index of surrounding media & $n_{1}=n_{2}$ & 1.0000 \\
\hline \hline
\end{tabular}

where $\mathcal{P}^{+} \mathcal{P}^{-}-\mathcal{Q}^{+} \mathcal{Q}^{-}=1$. In the absence of boundary reflections this reduces to $\mathcal{P}^{-}=0$, or $r_{c}, t_{c} \rightarrow \infty$, the established lasing condition for an index-matched DFB laser [11,19]. The lasing condition for a scalar DFB laser with boundary reflections can be determined from the last two rows of Eq. (3):

$$
\mathcal{P}^{-}+r_{1} \mathcal{Q}^{-}-r_{2} \mathcal{Q}^{+}-r_{1} r_{2} \mathcal{P}^{+}=0,
$$

which also reduces to $r_{c}, t_{c} \rightarrow \infty$ when $r_{1}=r_{2}=0$.

In order to determine where novel modes may occur, we can explicitly exclude $r_{c}, t_{c} \rightarrow \infty$, or $\mathcal{P}^{-} \rightarrow 0$, to find from Eq. (6) that

$$
\left[\begin{array}{lc}
r_{1} r_{2} e^{i k L} t_{c} & r_{1} r_{c} \\
e^{2 i(k+p) L} r_{1} r_{2}^{2} r_{c} & e^{i k L} r_{1} r_{2} t_{c}
\end{array}\right]\left[\begin{array}{l}
E_{L}^{+}(0) \\
E_{R}^{+}(0)
\end{array}\right]=\left[\begin{array}{c}
E_{L}^{+}(0) \\
E_{R}^{+}(0)
\end{array}\right] .
$$

Equation (9) is an eigenmode equation with matrix elements corresponding to the light paths in Fig. 2. It represents the replication of the propagating electric field every round-trip.

\section{RESULTS}

Given the real parameters $\kappa L, n_{1}, n_{2}$, and $n_{c}$, Eq. (7) can be solved numerically for the lasing modes in order to determine the complex detuning $\delta k L$. For a fixed distributed coupling strength $\kappa L=4$ and setting $r_{1}=r_{2}=0.278$ and $\lambda_{0}^{\mathrm{Br}}=530.4 \mathrm{~nm}$ (see Table I), we plot the absolute reciprocal of the left-hand side of Eq. (7) over a range of detuning $[\operatorname{Re}(\delta k L / 2)]$ and gain $[\operatorname{Im}(\delta k L / 2)]$ in Fig. 3, so diverging peaks correspond to lasing modes.

Within the range plotted, seven lasing peaks are observed that can be divided into a group with high-gain thresholds $\left(0_{L}, 1_{L}, 2_{L}, 3_{L}\right)$ and a group with low-gain thresholds $\left(1_{R}, 2_{R}, 3_{R}\right)$ (see Fig. 3 ). The labeling scheme relates to the chirality of the modes, as discussed below.

In order to determine the physical origin and characteristics of the modes, their movement is traced as the coupling strength $\kappa L$ is reduced to zero, as seen in Fig. 4. At zero coupling the only feedback mechanism is reflection from the boundaries, so the modes are Fabry-Pérot (FP) modes spaced $\Delta(\delta k L / 2)=\pi$ apart. It is seen that six of the modes split into paired branches from the degenerate FP modes and can thus be paired up as $\left(1_{L}, 1_{R}\right),\left(2_{L}, 2_{R}\right)$, and $\left(3_{L}, 3_{R}\right)$, leaving a lone $0_{L}$ mode. The dashed lines identify constant values of $\kappa L$.

Once a lasing mode has been identified, the forward propagating field at $z=0$ is determined from Eq. (6). While in general there are a number of eigenmode solutions to Eq. (6), we found that for the lasing modes, when $\kappa L \neq 0$,

$$
\left[\begin{array}{l}
E_{L}^{+}(0) \\
E_{R}^{+}(0)
\end{array}\right]=\left[\begin{array}{c}
-r_{1} \mathcal{Q}^{-} \\
\mathcal{P}^{-}-r_{1} r_{2} e^{i k L}
\end{array}\right] .
$$

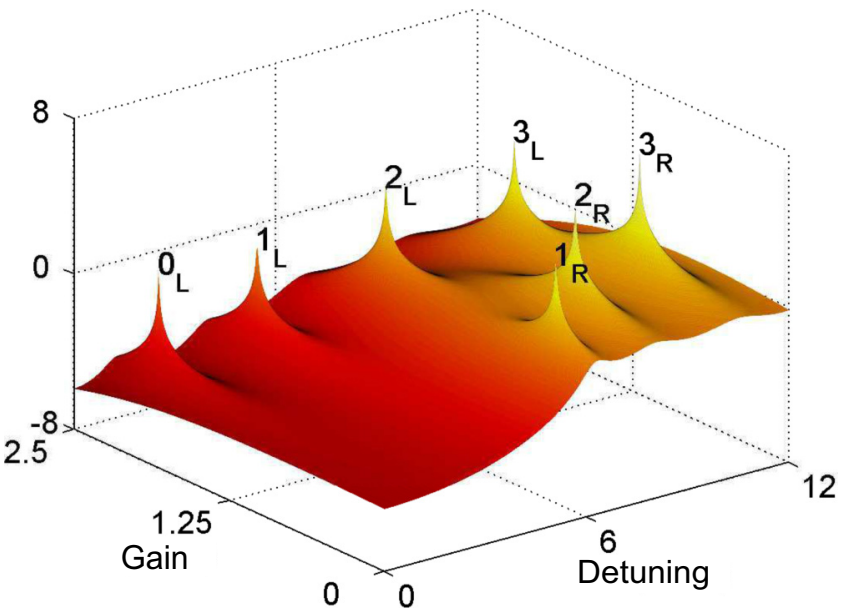

(a)

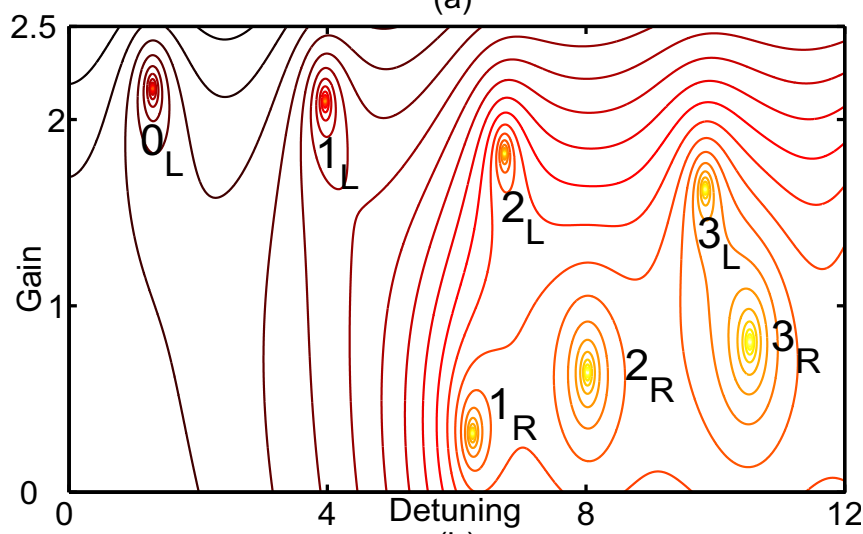

(b)

FIG. 3. (Color online) Lasing modes for a chiral laser as (a) a surface plot and (b) a contour plot. The lasing modes appear as poles that occur in two groups, one group with high-gain thresholds $\left(0_{L}, 1_{L}, 2_{L}, 3_{L}\right)$ and a group with low-gain thresholds $\left(1_{R}, 2_{R}, 3_{R}\right)$.

All lasing polarizations are elliptical, becoming more circular as $\kappa L$ increases. When $\kappa L=0$ the chiral laser becomes a

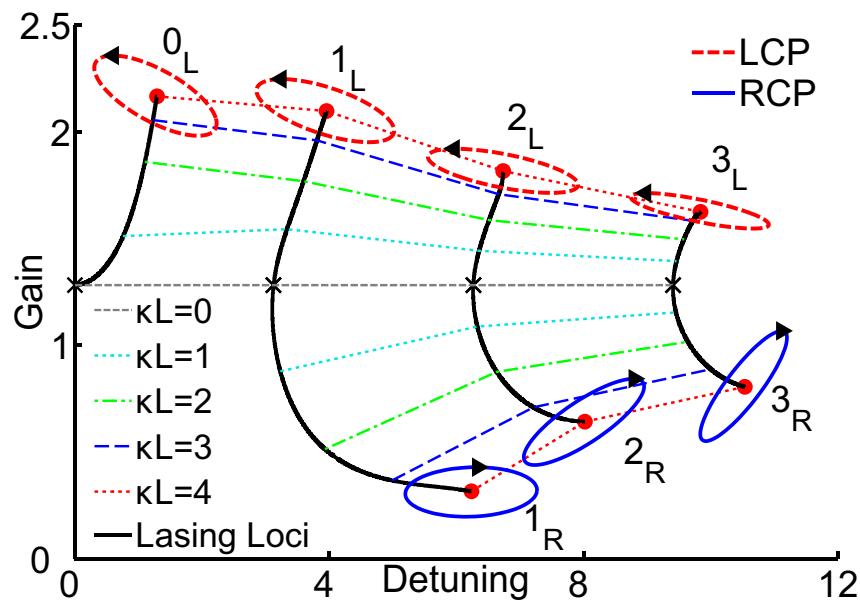

FIG. 4. (Color online) Movement of the lasing modes with respect to varying $\kappa L$. The solid lines trace the evolution of the modes of Fig. 3 (red dots) as $\kappa L$ is reduced to zero (black crosses). The dashed lines connect modes of constant $\kappa L$. The superimposed ellipses represent the lasing output polarization when $\kappa L=4$. 

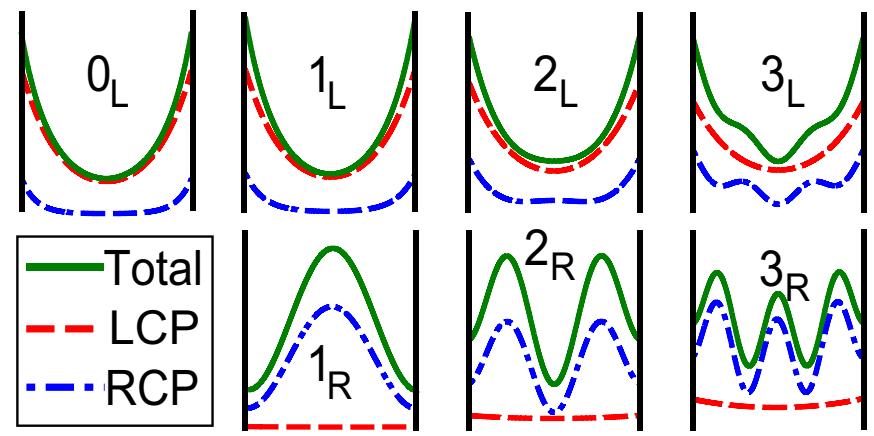

FIG. 5. (Color online) Intracavity intensity envelopes for the seven modes in Fig. 3 of the total (green solid line), LCP (red dashed line), and RCP (blue dash-dotted line) electric fields for a SCM cavity.

pure FP laser and the modes become polarization independent. The output polarization $\left[E_{L}^{+}(L), E_{R}^{+}(L)\right]^{T}$ is determined from Eq. (5). From Fig. 4 we note that the high-gain threshold modes lase LCP (dashed red ellipses) while the low-gain threshold modes lase RCP (solid blue ellipses).

In Fig. 5 we plot the total intensity envelope $I(z)=\left|E_{L}^{+}(z)\right|^{2}+\left|E_{R}^{-}(z)\right|^{2}+\left|E_{L}^{-}(z)\right|^{2}+\left|E_{R}^{+}(z)\right|^{2}$, as well as the LCP and RCP intensity envelopes $I_{L, R}(z)=$ $\left|E_{L, R}^{+}\right|^{2}+\left|E_{L, R}^{-}\right|^{2}$. The total energy of the higher modes is concentrated at the boundary, whereas for the lower modes the total energy is concentrated within the gain medium. In the higher modes RCP remains concentrated at the boundary and the FP mechanism generating LCP provides the majority of the laser power. The opposite is true for the lower modes, where the DFB mechanism generating
RCP dominates the FP mechanism generating LCP. There is therefore a clear distinction between the higher modes lasing LCP, which rely on the FP mechanism to lase, and the conventional, lower modes lasing RCP, which rely on the DFB mechanism [11].

The DFB mechanism in the lower modes allows lasing at a lower threshold than the degenerate FP modes at $\kappa L=0$. However, for the higher modes, intracavity LCP experiences a homogeneous medium, for which chirality reversing boundary reflections represent a loss mechanism into RCP. Introducing a right-handed SCM therefore increases the lasing threshold for the FP mechanism powering the high-gain threshold modes. When such a medium is introduced, the polarization degeneracy of the FP modes is lifted, so the modes bifurcate as shown in Fig. 4.

The lone status of the $0_{L}$ mode results from lasing within the photonic band gap for all $\kappa L$. For this mode the photon density of states is zero for RCP and it can therefore only lase via the FP mechanism. We see the intriguing possibility that a chiral laser can operate within the photonic band gap. The unique properties of the chiral laser ensure that this isolated mode occurs with opposite polarization to the principal modes of the DFB structure.

In Fig. 6 the boundary reflections are gradually reduced in order to examine the effect that decreasing the FP lasing mechanism has on the chiral lasing modes. We establish that as the boundary reflections approach zero the gain threshold for the higher modes diverges and only the lower modes remain. When the facet reflectivities are eliminated [Fig. 6(d)], the remaining modes agree with the scalar DFB modes enumerated by Kogelnik and Shank [11].
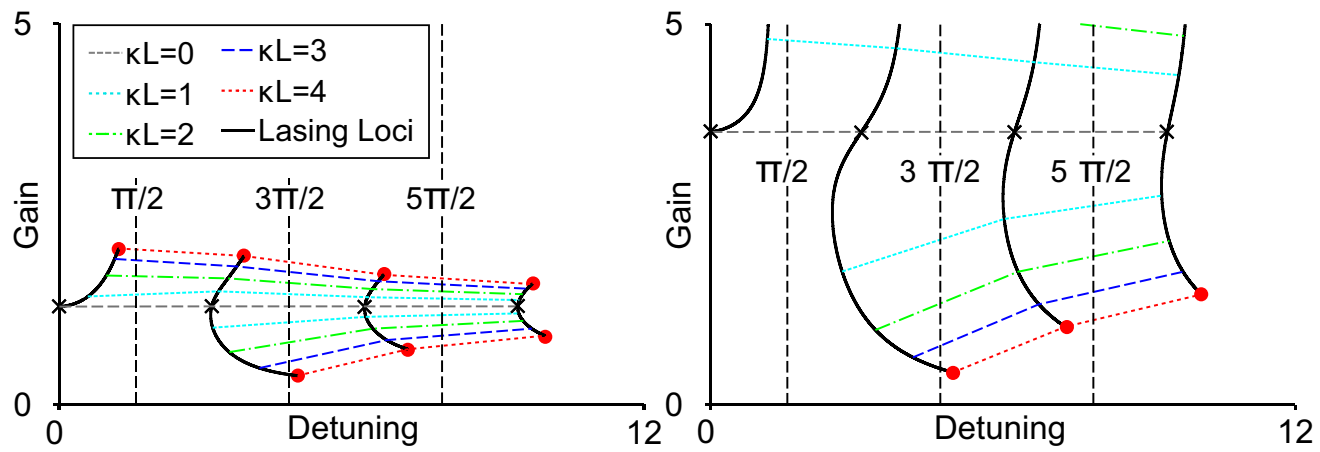

(a)

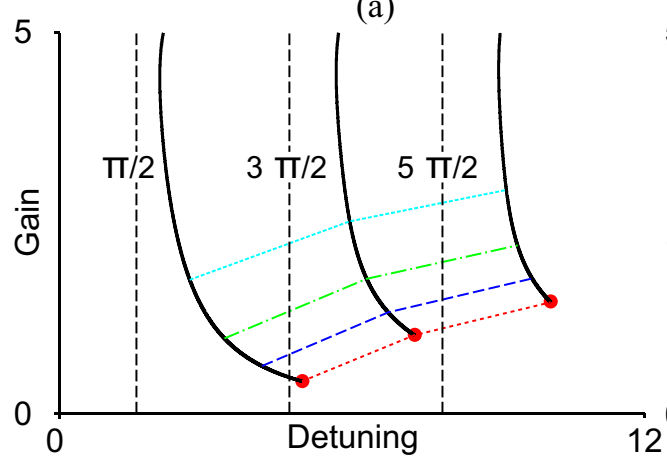

(c)

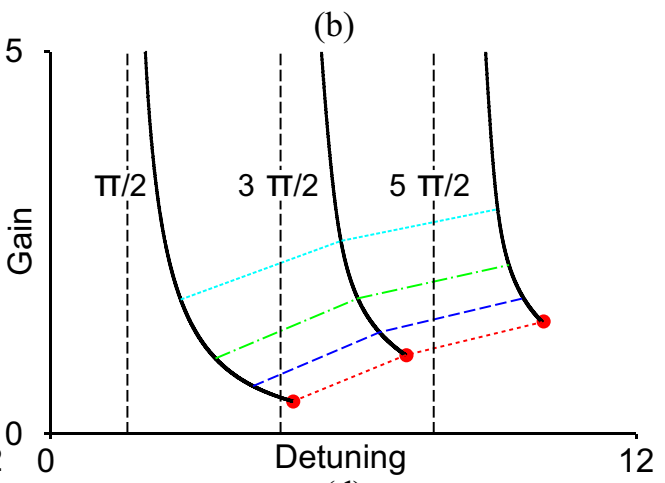

(d)

FIG. 6. (Color online) Evolution of chiral modes as the facet reflectivies are reduced by the given factor: (a) $r$, (b) $0.1 \times r$, (c) $0.01 \times r$, and (d) $0 \times r$. (a) corresponds to Fig. 4, while in (d) the purely RCP modes correspond exactly to the scalar DFB modes enumerated by Kogelnik and Shank [11]. 


\section{CONCLUSION}

This paper has derived the lasing condition for a chiral laser [Eq. (7)] and has characterized the modes in terms of their polarization as wave number and gain vary. We have shown that the mode structure of a chiral laser reflects the competition between the FP and DFB mechanisms. This suggests a simple means of achieving integrated polarization switching. One possible method involves modulating the frequency spectrum of the gain in order to target specific modes. Alternatively, tuning the laser modes into and out of the static gain spectrum would also allow for highly specific targeting of desired lasing modes. We have also shown that a chiral laser can lase within the photonic band gap.

The practical interest in using SCMs as laser hosts stems from their compact, simple construction [7,21], tunability via external stimuli [3], high slope efficiency, and circular polarization selectivity. They make good candidates for display technology [5], where their cavity length and polarization properties allow for higher resolutions than current lamp projectors. In spectroscopy [6] the broad tunability of a single source can be utilized to vary the penetration depth and for noninvasive medical treatment. The mode structure developed in this paper will facilitate continued development of these promising devices.

The analysis of this paper is restricted to the steady state. In further work, numerical integration of first-order pulse propagation equations [22,23] will enable a study of the dynamics, turn-on, and stability properties of chiral lasers. A local stability analysis of the lasing modes will prove particularly interesting, as it will demonstrate which of the lasing modes can realistically be used to lase. The methods employed in this paper can also be used to analyze a chiral laser in which there exists a twist defect [24]. Such a laser is the chiral analog of the $\lambda / 4$-shifted DFB laser, in which a very narrow defect mode exists at the center of the band gap. These topics will form the basis of future studies.

\section{ACKNOWLEDGMENT}

The authors wish to thank P. Kinsler for many helpful discussions and acknowledge support from the EPSRC.
[1] A. Beveratos, R. Brouri, T. Gacoin, A. Villing, J.-P. Poizat, and P. Grangier, Phys. Rev. Lett. 89, 187901 (2002).

[2] B. Mendiburu, Stereoscopic Digital Cinema from Script to Screen (Elsevier, Oxford, 2009).

[3] A. D. Ford, S. M. Morris, and H. J. Coles, Mater. Today 9, 36 (2006).

[4] A. Lakhtakia and M. W. McCall, Opt. Commun. 168, 457 (1999).

[5] S. M. Morris, P. J. W. Hands, S. Findeisen-Tandel, R. H. Cole, T. D. Wilkinson, and H. J. Coles, Opt. Express 16, 18827 (2008).

[6] H. Coles and S. Morris, Nat. Photon. 4, 676 (2010).

[7] K. Peddireddy, V. S. R. Jampani, S. Thutupalli, S. Herminghaus, C. Bahr, and I. Musevic, Opt. Express 21, 30233 (2013).

[8] D. J. Gardiner, S. M. Morris, P. J. W. Hands, C. Mowatt, R. Rutledge, T. D. Wilkinson, and H. J. Coles, Opt. Express 19, 2432 (2011).

[9] P. J. W. Hands, S. M. Morris, T. D. Wilkinson, and H. J. Coles, Opt. Lett. 33, 515 (2008).

[10] D. J. Gardiner, W.-K. Hsiao, S. M. Morris, P. J. W. Hands, T. D. Wilkinson, I. M. Hutchings, and H. J. Coles, Soft Matter 8, 9977 (2012).

[11] H. Kogelnik and C. V. Shank, J. Appl. Phys. 43, 2327 (1972).
[12] V. I. Kopp, B. Fan, H. K. M. Vithana, and A. Z. Genack, Opt. Lett. 23, 1707 (1998).

[13] J. P. Dowling, M. Scalora, M. J. Bloemer, and C. M. Bowden, J. Appl. Phys. 75, 1896 (1994).

[14] V. I. Kopp, A. Z. Genack, and Z.-Q. Zhang, Phys. Rev. Lett. 86, 1753 (2001).

[15] M. Pinol et al., Appl. Phys. Lett. 86, 051107 (2005).

[16] A. Lakhtakia and R. Messier, Sculptured Thin Films: Nanoengineered Morphology and Optics (SPIE, Bellingham, WA, 2005).

[17] P.-G. de Gennes and J. Prost, The Physics of Liquid Crystals (Oxford University Press, Oxford, 1995).

[18] M. W. McCall, J. Opt. A 11, 074006 (2009).

[19] A. Yariv, Quantum Electronics, 3rd ed. (Wiley, New York, 1989).

[20] G. P. Agrawal and N. K. Dutta, Long Wavelength Semiconductor Lasers (Van Nostrand Reinhold, New York, 1986).

[21] S. Furumi, Chem. Record 10, 394 (2010).

[22] P. Kinsler, Phys. Rev. A 81, 023808 (2010).

[23] P. Kinsler, Phys. Rev. A 81, 013819 (2010).

[24] I. J. Hodgkinson, Q. H. Wu, K. E. Thorn, A. Lakhtakia, and M. W. McCall, Opt. Commun. 184, 57 (2000). 